\title{
Antenna Model for Wire Lasers
}

\author{
E. E. Orlova* \\ Institute for Physics of Microstructures, Russian Academy of Sciences, Nishny Novgorod GSP-105, 603600, Russia \\ J. N. Hovenier, T. O. Klaassen, I. Kašalynas, ${ }^{\dagger}$ A. J. L. Adam, J. R. Gao ${ }^{\ddagger}{ }^{\circ}$ and T. M. Klapwijk \\ Kavli Institute of NanoScience Delft, Faculty of Applied Sciences, Delft University of Technology, \\ Lorentzweg 1, 2628 CJ, Delft, The Netherlands \\ B. S. Williams, S. Kumar, and Q. Hu \\ Department of Electrical Engineering and Computer Science and Research Laboratory of Electronics, \\ Massachusetts Institute of Technology, Cambridge Massachusetts 02139, USA \\ J. L. Reno \\ Sandia National Laboratories, Albuquerque, New Mexico 87185-0601, USA
}

(Received 7 December 2005; published 5 May 2006)

\begin{abstract}
An antenna model is proposed for long $(L \gg \lambda)$ lasers with subwavelength cross sections (wire lasers). It is shown that the far-field pattern of the wire lasers is determined by the ratio of the wavelength to the length. The radiation of the wire laser is predicted to be concentrated in a narrow beam $\Theta \simeq \sqrt{2 \lambda / L}$ for laser modes where the longitudinal phase velocity is in synchronism with the velocity of light in air. Experimental results obtained using a terahertz quantum cascade wire laser are in agreement with the model.
\end{abstract}

DOI: 10.1103/PhysRevLett.96.173904

PACS numbers: 42.60.Jf, 42.25.-p, 78.67.-n

Is it possible to concentrate radiation from a laser with a subwavelength cross section into a narrow beam? The combination of a small laser aperture with a low beam divergence would open the perspective of local laser excitation of individual nano-objects, which would be of interest in numerous applications such as optical communication, high-density magneto-optic data storage, biological studies, and quantum information. However, the reduction of a laser aperture is known to cause an increase of the beam divergence due to diffraction. The diffraction limited minimum angular size of the beam for radiation with a wavelength $\lambda$ for an aperture of size $a$ is determined by $\Theta \simeq \lambda / a$ for sources with $a>\lambda$ [1]. Thus, for example, in diode lasers, the localization of the optical mode in a thin active region (with the width of the order of several wavelengths) leads to a high beam divergence in the plane perpendicular to the active layer [2]. High efficiency and gain achieved in nanostructure lasers permits laser dimensions comparable to or smaller than the wavelength [3-5]. Highly divergent radiation is expected from lasers with subwavelength apertures [4]. The methods used to improve the directivity of laser radiation, using, for example, surface emission [2], an array of lasers [6], or using external optical elements, are all based on the increase of the effective size of an aperture. The recently discovered effects of surface plasmons on the transmission of light through subwavelength apertures [7] also imply an increase of the effective aperture size due to the formation of plasmon-polaron excitations at the surface of a metallic screen. The development of nanotechnologies has brought along new methods to manipulate light on the scales com- parable to the wavelength using the concepts of photonic crystals [8], left-handed synthetic materials [9], and onedimensional plasmonic waveguides [10]. These methods however do not solve the problem of the guidance of light in the air outside the artificially fabricated media.

In this Letter we propose a method to achieve a high directivity for lasers with subwavelength apertures (wire lasers). The idea is based on an antenna approach to analyze laser modes. Since individual sources in the laser medium emit coherently with their phases determined by the cavity modes, each laser mode can be thought of as a continuous phased array. The different laser modes do not interfere because of their slightly different frequencies and the far field of a laser operating in a multimode regime is therefore a combination of intensities provided by different modes, as if there are several phased arrays at the same location. We show that wire lasers with subwavelength transverse dimensions and with lengths much bigger than the wavelength $(L \gg \lambda)$ are analogous to traveling wave antennas. Their far field is determined by the interference of radiation from the longitudinal distribution of emission sources. The beam pattern of the wire lasers consists of axially symmetric conelike maxima with angular sizes determined by the ratio of the wavelength to the length of the laser cavity. The predictions of the model have been verified experimentally using wire lasers based on terahertz quantum cascade structures. The short range axially symmetric variations of the far-field intensity have been observed with the angular sizes determined by the length of the laser in agreement with our model. We predict that the synchronization of the longitudinal phase velocities of the 
modes with that of light in air will produce a concentration of the laser radiation in a narrow beam due to the interference of radiation from the longitudinal mode distribution. The angular divergence of such a beam is determined by the square root of the wavelength divided by the length of the laser cavity: $\Theta \simeq \sqrt{2 \lambda / L}$. Hence, there are no principal limitations on the beam divergence of the wire laser due to the small aperture size: a laser beam of any desired directivity can be obtained by making the laser longer.

The calculation of the beam profile from a wire laser cannot be performed using the standard aperture diffraction methods [11]. These methods, based on Huygens' principle, are rigorous when applied to the field distribution on a closed surface. Approximate solutions are obtained using the integration over the surface of the aperture. The frequently used method of the Kirchhoff integral over the aperture with a finite size leads to solutions inconsistent with Maxwell equations. It gives suitable approximations of the beam pattern only for large apertures and paraxial diffraction angles [12]. The electrodynamic formulations of Huygens' principle are based on the vector Green theorem [13], Lorentz lemma [14], and uniqueness theorem [15] providing solutions of radiation problems consistent with Maxwell equations. However in the case of subwavelength apertures the beam pattern is influenced significantly by the field distribution outside the aperture. Extension of the surface of integration to the infinite plane perpendicular to the longitudinal axis of the laser beyond the borders of the aperture would allow to account for this influence. Unfortunately, the exact calculation of the near field beyond the borders of the aperture is a complicated problem and it should be noted that approximate methods lead to large relative errors in regions where the field values are small. Besides, it should also be noted that the laser modes are often calculated without taking into account the influence of the length of the laser on the transverse field distribution. As will be shown, the latter turns out to be of primary importance for the far field of wire lasers.

The problem of calculating the transverse field distribution outside the laser can be avoided using an alternative approach based on the equivalence of displacement currents in dielectric and conductivity currents. The radiation field can then be expressed in terms of the field values inside the volume of the cavity. The latter are relatively big and thus are easier to determine and verify. This method naturally allows us to account for and analyze the influence of the laser length on the beam structure. It can be shown [16] that the system of Maxwell equations for radiation in a dielectric medium is equivalent to that for radiation in vacuum with equivalent current sources with the complex amplitudes (Gaussian units):

$$
\mathbf{J}_{\text {eq }}=\mathbf{J}_{c}+\frac{j \omega(\varepsilon-1)}{4 \pi} \mathbf{E},
$$

where $\mathbf{J}_{c}$ is the conduction current distribution, $\mathbf{E}$ the electric field, $\varepsilon$ the dielectric constant, and $\omega$ the frequency of the field. The field radiated by the laser is the same as that of the equivalent current distribution and is described by the electric vector potential:

$$
\mathbf{A}=\frac{1}{c} \int_{v} \mathbf{J}_{\mathrm{eq}} \frac{\exp (-j k r)}{r} d V,
$$

where the integration is carried out over the volume of the laser, with $k=\omega / c, c$ the velocity of light, and $r$ the distance between the observation point and the arbitrary point inside the laser. In the Lorentz gauge, the electric field is expressed in terms of the vector potential as

$$
\mathbf{E}=-\frac{j \omega}{c} \mathbf{A}+\frac{c}{j \omega} \boldsymbol{\nabla}(\boldsymbol{\nabla} \cdot \mathbf{A}) .
$$

To calculate the field outside the source differential operations of Eq. (2) can be introduced in the integral. In the far field the expression for the electric field can be simplified further by using for the phase factor $k r \simeq k R-\left(\mathbf{k} \cdot \mathbf{r}^{\prime}\right)$, with $\mathbf{R}$ the radius vector from the reference point inside the laser cavity to the observation point, $\mathbf{r}^{\prime}$ the radius vector of the integration point of the laser cavity, and $\mathbf{k}=k \mathbf{R} / R$, and using $r \simeq R$ for the denominator of Eq. (2):

$$
\mathbf{E}=-\frac{j \omega}{c^{2}} \frac{\exp (-j k R)}{R} \sum_{i} \mathbf{E}_{i} \int_{v} J_{\mathrm{eq}}^{i} \exp \left(j \mathbf{k} \cdot \mathbf{r}^{\prime}\right) \mathbf{d} \mathbf{r}^{\prime}
$$

The term $\mathbf{E}_{i}=\left[\mathbf{r}_{0} \times\left(\mathbf{x}_{0}^{i} \times \mathbf{r}_{0}\right)\right]$ is the polarization factor of the electric field produced by the component of the current along the $i$ coordinate axis, $\mathbf{r}_{0}=\mathbf{R} / R, \mathbf{x}_{0}^{i}$ is the unit vector in the $i$ direction, and $J_{\text {eq }}^{i}$ are the Cartesian components of the equivalent current vector.

At this point we have to make an assumption about the structure of the laser mode. We assume that the longitudinal dimension of the laser is much larger than the transverse dimensions $(L \gg a)$ and than the wavelength $(L \gg \lambda)$. Let the laser medium be invariant to inversion and periodic in the longitudinal direction with period $\zeta$ much smaller than the wavelength. Solutions of the wave equation for an infinite medium periodic in the $z$ direction are Bloch functions in the $z$ coordinate. Thus the equivalent current for an optical mode can be presented as a linear combination of Bloch functions:

$$
J_{\mathrm{eq}}^{i}=\tilde{J}^{i}(\rho, z)[\exp (j q z) \pm \exp (-j q z)],
$$

where $\rho$ is the transverse radius vector, $\tilde{J}^{i}(\rho, z)$ the periodic function of the longitudinal coordinate $z$, and the wave number of the mode $q$ is a function of the mode frequency $\omega$ and transverse field distribution of the mode. Because of the inversion symmetry the equivalent currents, $J_{\text {eq }}^{i}$, are either even [the plus sign in Eq. (5)] or odd (the minus sign) parity functions of the $z$ coordinate. The longitudinal phase velocity is introduced as $c_{L}=\omega / q$. Boundary conditions at the ends of the laser wire $(z= \pm L / 2)$ determine a discrete frequency spectrum for laser modes. A combination of Bloch waves does in general not satisfy the boundary conditions, the edges of a wire laser can be considered as sources of spherical and evanescent waves. We assume that the equivalent current inside the laser with finite length 
is described by Eq. (5), neglecting the edge effects in view of our assumption of a large laser length. The integrals in Eq. (4) with the current sources given by Eq. (5) can be approximated by averaging the fast oscillating functions over the period $\frac{1}{\zeta} \int_{\zeta} \tilde{J}^{i}(\rho, z) d z=\bar{J}^{i}(\rho)$. Then the integrals over the longitudinal and the transverse coordinates in Eq. (4) factor out and the far-field amplitude is given by

$$
\mathbf{E}=-\frac{j \omega}{c^{2}} \frac{\exp (-j k R)}{R} L \sum_{i} \mathbf{E}_{i} F_{i}^{\perp} F_{i}^{z}
$$

where

$$
F_{i}^{\perp}=\int_{S} \bar{J}^{i}(\rho) \exp \left(j \mathbf{k}_{\perp} \cdot \rho\right) \mathbf{d} \rho
$$

is an integral over the transverse field distribution analogous to that used in aperture methods of the far-field calculation [1]. When the aperture size is larger than the wavelength, this transverse factor determines the minimum angular divergence of the beam formed by the interference of radiation from the transverse distribution of emission sources:

$$
\Theta \simeq \lambda / a .
$$

In the case of an aperture much smaller than the wavelength $k a \ll 2 \pi$, the exponential term in Eq. (7) can be expressed as a power series of $k_{i} \rho_{i}$, where $k_{i}$ and $\rho_{i}$ are the components of the wave vector and the transverse radius vector. The angular dependence of the transverse factor is determined by $k_{i}^{m} k_{j}^{l}$ corresponding to the first nonvanishing momentum of the transverse distribution of the equivalent current, which gives a slow angular dependence or no

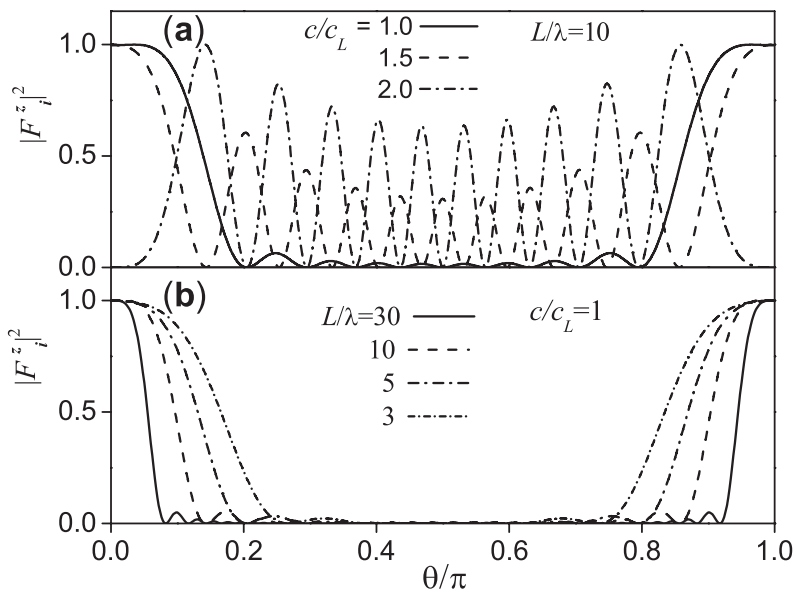

FIG. 1. The dependence of the normalized far-field intensity determined by the square of the longitudinal factor $\left|F_{i}^{z}\right|^{2}$ on the angle with respect to the axis of the wire laser (a) for different ratios of the longitudinal phase velocity of the laser mode $c_{L}$ to the velocity of light $c$. (b) for different ratios of the laser length $L$ to the wavelength $\lambda$ in the case of the phase synchronism. Note the two main lobes in the case of the phase synchronism $\left(c_{L} / c=\right.$ 1) in the forward direction and backward direction along the laser axis with the width $\Theta \simeq \sqrt{2 \lambda / L}$. dependence at all (when both $m$ and $n$ are equal to zero). In this case the far-field amplitude is determined mainly by the longitudinal factor (Fig. 1):

$$
F_{i}^{z}=\frac{\sin \left(\varphi_{+}\right)}{\varphi_{+}} \mp \frac{\sin \left(\varphi_{-}\right)}{\varphi_{-}},
$$

where $\varphi_{ \pm}=\left[c / c_{L} \pm \cos (\theta)\right] \pi L / \lambda$ are the phase differences for the radiation associated with $\pm q$ components of $J_{\mathrm{eq}}^{i}$, coming from the center $(z=0)$ and from the edges $(z= \pm L / 2)$ of the laser, $\theta$ is an angle between the longitudinal axis and the direction to the observation point. The minus and the plus signs in Eq. (9) are used for even and odd $J_{\text {eq }}^{i}$ respectively. The components of the longitudinal factor introduced in Eq. (9) are analogous to the far field of traveling wave antennas. The pattern is axially symmetric with maximum amplitudes in the directions $\theta$ given by the relation

$$
\left|\varphi_{ \pm}\right|=\left|\left[c / c_{L} \pm \cos (\theta)\right] \pi L / \lambda\right|=0, \quad(n+1 / 2) \pi,
$$

with $n$ an integer.

For velocities $c_{L}<c$, typical for a semiconductor medium, there is no direction in which all the sources along the cavity emit in phase $\left(\left|\varphi_{ \pm}\right|>0\right)$. The maxima correspond to directions where the number of sources emitting in phase is relatively big, and form circles in the plane perpendicular to the longitudinal axis [Figs. 2(a) and 2(b)]. The angular size $d \theta$ of the intensity variations is deter-
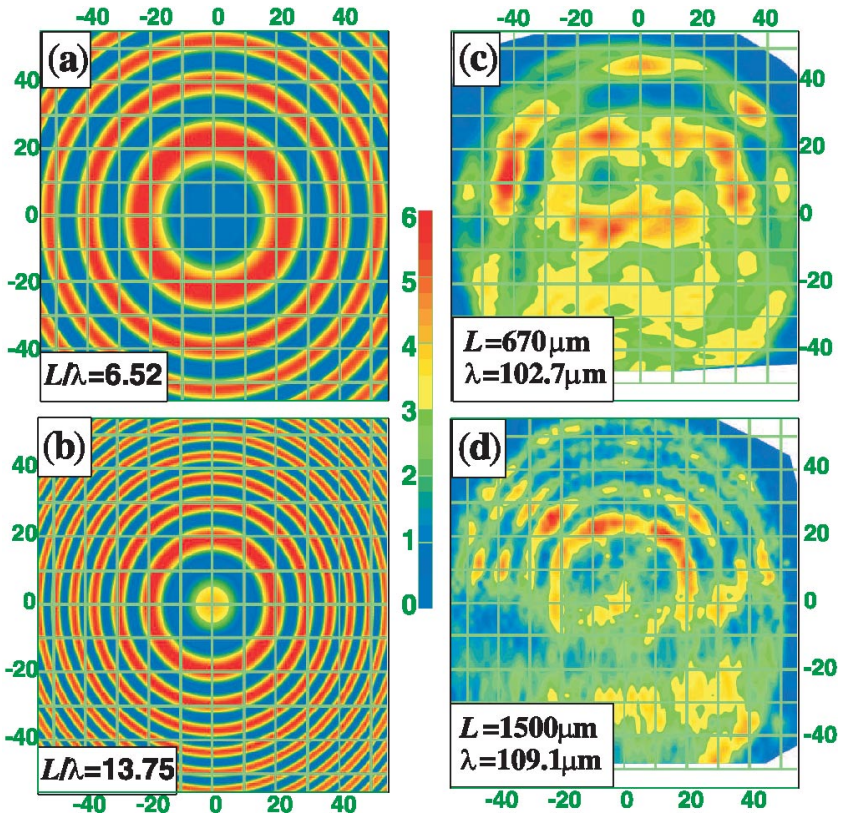

FIG. 2 (color). (a)-(b) Calculated angular dependence of the far-field intensity in the plane perpendicular to the longitudinal axis of the laser for $c / c_{L}=3.3$ and different ratios of the length $L$ and the wavelength $\lambda$ of the laser. An "equirectangular projection" has been used; scales are given in degrees. (c)(d) experimental beam patterns from terahertz QCL's with the same $L / \lambda$ ratios. Note the circular structure; the angular width of the circles decreases with the increase of $L / \lambda$. 
mined by the ratio of the wavelength to the laser length: $\cos (\theta+d \theta)-\cos (\theta)=\lambda / L$. The decay of the intensity from one circle to another is slower for larger differences in phase velocities.

In the specific case of synchronism of the longitudinal phase velocity with that of light $c_{L}=c$, only two strong lobes (see Fig. 1) are formed in the positive and negative directions of the longitudinal axis where all the sources emit in phase $\left(\varphi_{ \pm}=0\right)$. The width of these lobes is determined by the relation $\varphi_{ \pm}=\pi$, which corresponds to the condition that the phase difference for radiation from all the sources along the laser length is smaller than $2 \pi$. From Eq. (10), in the limit of small angle $1-\cos (\theta) \simeq$ $\theta^{2} / 2$, we have

$$
\Theta \simeq \sqrt{2 \lambda / L} .
$$

This angle is smaller than the diffraction angle associated with the transverse factor [Eq. (8)], and determines the divergence of the beam when the Fresnel number of the laser is small: $N_{F}=a^{2} / \lambda L \ll 1$, which is always true for subwavelength wire lasers.

This far-field pattern is independent of the mechanism of laser emission and is general for all wire lasers. Wire lasers can be based on diode structures, quantum wires, arrays of quantum dots, etc., We have tested our model for $c / c_{L} \approx$ 3.3 using single mode terahertz $(\lambda \simeq 100 \mu \mathrm{m})$ quantum cascade lasers (QCL's) formed by GaAs based structures with subwavelength apertures $10 \mu \mathrm{m} \times 25 \mu \mathrm{m}$ and metallic contacts ("metal-metal waveguide" [4,17]). The length is much larger than the wavelength. Details of the experimental study of the beam profile of the cascade lasers are presented elsewhere [18]. The observed far-field beam pattern shows strong modulations of the intensity [Figs. 2(c) and 2(d)]. The beam profile is almost axially symmetric with the directions of the maxima forming cones around the longitudinal axis of the waveguide in agreement with our model. Relatively low intensity is experimentally observed in the lower part of the plane perpendicular to the longitudinal axis, because in that part of space the radiation from the cavity is partially blocked by the sample holder. The angular widths of the maxima decrease with the increase of the cavity length. The directions of the maxima are well described by Eq. (10). We conclude that the experimental results are in very good agreement with the model.

The experimental verification of our prediction of high directivity for wire lasers with a suitable value for the phase velocities $c_{L}=c$ is a matter of future investigations. Different solutions can be found to achieve the synchronism depending on the type of a laser. For example, for a dielectric waveguide with metal contacts the laser modes near the cutoff regime are known to have longitudinal phase velocities close to that of light.

In conclusion, we have proposed an antenna model for long lasers with subwavelength apertures and verified some predictions experimentally. Based on this model we show that the far-field pattern of wire lasers is determined by the ratio of the wavelength to the length of the laser. We predict that in case of synchronism of the longitudinal phase velocity of the optical mode with that of light the radiation can be concentrated in a narrow beam with a divergency below the diffraction limit.

We acknowledge constructive and helpful discussions with E. F. Orlov, A. M. Belyantsev, N.F. Kovalev, A. Baryshev, J. P. Woerdman, J. J. M. Braat, H. P. Urbach, and R. A. Suris. E. E. Orlova gratefully acknowledges the NATO and NWO visiting grants, and RFBR Grant No. 0602-17437a. I. Kašalynas and A. J. L. Adam acknowledge support through the European Union HP-RTN Project under Contract No. HPRN-CT-2002-00206. The work at MIT is supported by AFOSR, NASA, and the NSF. Sandia is a multiprogram laboratory operated by Sandia Corporation, a Lockheed Martin Company, for the U.S. Department of Energy under Contract No. DE-AC0494AL85000.

*Electronic address: orlova@ipm.sci-nnov.ru

${ }^{\dagger}$ Permanent address: Semiconductors Physics Institute, A. Gostauto 11, LT-01108 Vilnius, Lithuania.

${ }^{\ddagger}$ Also with SRON National Institute for Space Research, Utrecht, The Netherlands.

[1] M. Born and E. Wolf, Principles of Optics: Electromagnetic Theory of Propagation, Interference and Diffraction of Light (Pergamon, Oxford, 1999), 7th ed.

[2] Zh. I. Alferov et al., IEEE J. Quantum Electron. 11, 449 (1975).

[3] R. Kohler et al., Nature (London) 417, 156 (2002).

[4] S. Kohen, B. S. Williams, and Qing Hu, J. Appl. Phys. 97, 053106 (2005).

[5] K. J. Vahala, Nature (London) 424, 839 (2003).

[6] R. Waarts, D. Mehuys, D. Nam, D. Welch, W. Streifer, and D. Scifres, Appl. Phys. Lett. 58, 2586 (1991).

[7] W. L. Barnes, A. Dereux, and T.W. Ebbesen, Nature (London) 424, 824 (2003).

[8] Yablonovitch, Phys. Rev. Lett. 58, 2059 (1987); S. John, Phys. Rev. Lett. 58, 2486 (1987).

[9] D. R. Smith, J. B. Pendry, and M. C. K. Wiltshire, Science 305, 788 (2004).

[10] Junichi Takahara et al., Opt. Lett. 22, 475 (1997); Aristeidis Karalis et al., Phys. Rev. Lett. 95, 063901 (2005).

[11] S. Silver, Microwave Antenna Theory and Design (Peregrinus, London, 1984).

[12] J. D. Jackson, Electrodynamics (Wiley, New York, 1998), 3rd ed.

[13] J. A. Stratton and L. I. Chu, Phys. Rev. 56, 99 (1939).

[14] A.Z. Fradin, Antennas of Ultra-High Frequencies (Soviet Radio, Moscow, 1957).

[15] S. A. Schelkunoff, Phys. Rev. 56, 308 (1939).

[16] Computer Techniques for Electromagnetics, edited by R. Mitra (Pergamon, Elmsford, NY, 1973), Chap 2.

[17] Q. Hu et al., Semicond. Sci. Technol. 20, S228 (2005).

[18] A. J. L. Adam et al., Appl. Phys. Lett. 88, 151105 (2006). 